\title{
СВЯЗЬ ЭМОЦИОНАЛЬНОГО ИНТЕЛЛЕКТА И ЛИЧНОСТНЫХ ЧЕРТ С НАСТРОЕНИЕМ
}

\author{
Д.В. ЛЮСИН, В.В. ОВСЯННИКОВА
}

\begin{abstract}
Резюме
Основная цель исследования состояла в поиске связей настроения с личностными чертами и эмоциональным интеллектом (ЭИ). Дополнительная цель состояла в получении новых психометрических данных по использованным методикам. Для измерения настроения использовалась методика ШПАНА, являющаяся русской адаптацией методики PANAS и оценивающая настроение по шкалам позитивного аффекта (ПА) и негативного аффекта (НА). Личностные черты измерялись с помощью опросника Большая Пятерка, эмоциональный интеллект - с помощью опросника ЭмИн. Методики проводились на выборке, состоявшей из 71 испытуемого в возрасте от 18 до 49 лет. Получены данные о внутренней согласованности шкал всех использованных методик, которая в основном является удовлетворительной $-\alpha$ Кронбаха всех шкал за одним исключением лежит в диапазоне от 0.78 до 0.89. Получены положительные корреляции ряда шкал опросника ЭмИн (общий ЭИ, внутриличностный ЭИ, Управление эмоциями) со шкалой ПА и отрицательные корреляции со шкалой НА. Шкалы межличностного ЭИ и Понимание эмоций дали только положительные корреляции со шкалой ПА. Получена отрицательная связь нейротизма со шкалой ПА и положительная связь со шкалой НА. Экстраверсия положительно связана со шкалой ПА и не имеет связей со шкалой НА. В совокупности полученные результаты соответствуют результатам аналогичных исследований, проводимых в других странах. Обосновывается необходимость в дальнейшем перейти от одноразовых замеров настроения к сбору данных о настроении испытуемых в течение длительного времени (так называемый experience sampling). Это позволит получить более достоверные сведения об эмоциональном фоне, характерном для того или иного испытуемого, и проанализировать связи между личностными чертами и эмоциональным интеллектом, с одной стороны, и динамическими характеристиками эмоциональных состояний, с другой стороны.
\end{abstract}

Ключевые слова: настроение, эмоциональный интеллект, Большая Пятерка, личностные черты.

Исследование выполнено в рамках Программы фундаментальных исследований НИУ ВШЭ в 2015 г. и при финансовой поддержке Научного фонда НИУ ВШЭ в 2014 г. по проекту № 14-09-0197. 
Настроение является одним из наиболее широко изучаемых типов эмоциональных явлений. Традиционно в психологии эмоций отмечается, как трудно поддаются определению те или иные эмоциональные явления, не говоря уже о самой категории «эмоция». Настроение в этом смысле является счастливым исключением. Различные авторы примерно одинаково трактуют это понятие, расходясь только в акцентах. Под настроением понимается длительное эмоциональное состояние, как правило, невысокой интенсивности (в норме) и не имеющее выраженного предметного характера (например: Рубинштейн, 1946; Frijda, 1993). Под последней характеристикой имеется в виду то, что настроение либо переживается как вообще беспредметное (т.е. субъективно человек не связывает его с конкретными событиями), либо возникает по поводу каких-то произошедших или ожидаемых событий, но легко «отделяется» от них и продолжает существовать в качестве эмоционального фона дальнейшей деятельности. Особое внимание исследователей привлекают расстройства настроения, так как они являются наиболее часто встречающимся видом психических расстройств. Нормальное настроение также представляет большой интерес для изучения, потому что оно влияет на любые психические процессы, включая когнитивные, будучи само в высокой степени подвижным и постоянно меняющимся под влиянием широкого круга воздействий.

Настроение обычно описывается с помощью многомерных моделей, выделяющих некоторое количество шкал, по которым характеризуются эмоциональные состояния человека. Сфера применения многомерных моделей эмоций весьма широка, при этом в исследованиях настроений чаще всего используются именно они. В последние десятилетия основными конкурирующими типами моделей являются трехмерные и двухмерные. Исторически наиболее ранняя и при этом широко известная модель была предложена В. Вундтом, который выделяет измерение, связанное с валентностью, а также измерения возбуждения и напряжения (Wundt, 1896). Примечательно, что похожие модели получаются и у современных авторов, использующих совершенно иную методологию эмпирических исследований (Schimmack, Grob, 2000; Люсин, 2014). Одной из наиболее известных двухмерных моделей является круговая модель Л. Фельдман-Барретт и Дж. Расселла (Feldman Barrett, Russell, 1999). В ней выделяются биполярные измерения валентности и напряжения. Ей противостоит другая известная двухмерная модель, предложенная Д. Уотсоном с соавт. (Watson et al., 1988), в которой выделяется две униполярные шкалы: позитивного аффекта (ПА) и негативного аффекта (НА). В каждой из этих шкал объединяются валентность и активация. Высоким баллам по шкале ПА соответствуют положительные эмоции с высокой активацией, а высоким баллам по шкале НА отрицательные эмоции с высокой активацией. Особенностью модели Уотсона с соавт. является трактовка положительного и отрицательного аффекта не как двух взаимоисключающих полюсов, а как ортогональных 
измерений. Это дает возможность описывать эмоциональные состояния смешанной валентности, т.е. переживаемые как положительные и отрицательные одновременно. На основе этой модели авторами была создана методика PANAS - Positive Affect Negative Affect Schedule. Несмотря на использование термина «аффект» в названии шкал и методики в целом, она используется ее разработчиками и другими исследователями для измерения именно настроения. Методика PANAS отличается высоким психометрическим качеством и широко используется в разнообразных исследованиях эмоций.

Факторы, предопределяющие настроение, очень разнообразны. Их можно разделить на внешние и внутренние, т.е. относящиеся непосредственно к субъекту. Такое различение носит условный характер, потому что внешние воздействия всегда преломляются через восприятие и оценки субъекта, однако оно полезно, так как позволяет структурировать данную область исследований. В настоящей работе нас интересуют те внутренние факторы настроения, которые являются устойчивыми характеристиками субъекта; они относительно стабильны во времени и устойчивы по отношению к отдельным ситуативным воздействиям. В качестве таких факторов были выбраны личностные черты, входящие в модель «Большая Пятерка» (McCrae, Costa, 2005), и эмоциональный интеллект.

Многие авторы отмечают, что личностные черты, описывающиеся моделью «Большая Пятерка», в существенной степени предопреде- ляют аффективные реакции людей (David et al., 1997). Например, люди c высоким нейротизмом склонны испытывать широкий спектр отрицательных эмоции, таких как грусть, тревога, злость, а экстраверсия и сознательность, напротив, отрицательно коррелируют с переживанием грусти (Velting, 1999). Люди с высокими баллами по шкале сознательности склонны к переживаниям положительных эмоций (Watson, Clark, 1992). Связи такого рода часто объясняются и на теоретическом, и на эмпирическом уровне особенностями эмоциональной регуляции людей с теми или иными личностными чертами.

Роль эмоционального интеллекта в настроении человека обнаруживается чаще всего в тех исследованиях, где эмоциональный интеллект измеряется с помощью опросников. Так, по данным Н. Шутте, более высокий эмоциональный интеллект связан с преобладанием положительного настроения, при этом связей с отрицательным настроением выявлено не было (Schutte et al., 2002). Heсколько исследований было выполнено с применением опросника TMMS - Trait Meta-Mood Scale (Salovey et al., 1995). В него входят шкалы внимания к своим эмоциям, ясности эмоций (насколько ясно в сознании человека представлены его эмоциональные переживания) и восстановления (насколько успешно человеку удается останавливать переживание своих отрицательных эмоций и поддерживать положительные эмоции). Было показано, что высокие баллы по шкале восстановления и низкие баллы по шкале внимания связаны с положительным 
настроением (Там же). В других исследованиях шкалы ясности и восстановления давали прямые корреляции с положительным настроением и обратные - с отрицательным (Palmer et al., 2002; Extremera, Fernández-Berrocal, 2005; FernándezBerrocal, Extremera, 2006).

Настоящее исследование имеет поисковый характер и направлено на выявление связей между настроением, с одной стороны, и личностными чертами и эмоциональным интеллектом, с другой стороны. Для измерения настроения использовалась методика ШПАНА, созданная Е.Н. Осиным и являющаяся русской адаптацией PANAS (Осин, 2012). Эта адаптация характеризуется высоким психометрическим качеством, автором показана сопоставимость русской и английской версий на уровне эквивалентности единицы измерения. Побочная цель исследования состояла в получении новых данных о надежности и валидности методики ШПАНА, которая является полезным инструментов для изучения эмоций на выборках русскоязычных испытуемых. Автор методики ШПАНА приводит данные о ее связях с некоторыми эмоциональными личностными чертами: шкала ПА положительно коррелирует с удовлетворенностью жизнью, субъективным счастьем и оптимизмом, а шкала НА отрицательно коррелирует с теми же переменными. Получен также ряд логичных связей с мотивационными переменными, однако данных о связях с факторами «Большой Пятерки» и эмоциональным интеллектом пока не существует. Получение таких данных нам представляет важным по двум при- чинам. Во-первых, национальные адаптации психологических методик всегда имеют некоторую специфику, поэтому важно изучать их психометрические свойства, в том числе их связи с другими методиками, измеряющими разнообразные индивидуально-психологические характеристики. Во-вторых, связи между эмоциональными состояниями человека и его устойчивыми личностными свойствами могут иметь культурную специфику, так как культура в значительной степени детерминирует особенности переживаний эмоций, правила их выражения и регуляции, степень социальной приемлемости (Берри и др., 2007).

Мы предположили, что шкала ПА будет положительно связана с эмоциональным интеллектом и с экстраверсией, а шкала НА - отрицательно. Для нейротизма мы ожидали противоположный паттерн связей.

\section{Метод}

Испытуемые. В исследовании принял участие 71 человек в возрасте от 18 до 49 лет $(\mathrm{M}=24.5, \mathrm{SD}=6.2)$, из них 52 женщины (73\%). Испытуемые - представители различных профессий и студенты.

Измерительные методики. Для оценки настроения использовалась методика ШПАНА (Осин, 2012), описанная выше. Она состоит из 20 пунктов и дает оценки настроения по двум шкалам - положительный аффект (ПА) и отрицательный аффект (HA).

Эмоциональный интеллект измерялся с помощью опросника ЭмИн (Люсин, 2009). Этот опросник состоит из 46 пунктов, имеет четыре 
основные шкалы - межличностный эмоциональный интеллект (МЭИ), внутриличностный эмоциональный интеллект (ВЭИ), понимание эмоций (ПЭ), управление эмоциями (УЭ), их объединение дает обобщенную оценку эмоционального интеллекта (ОЭИ).

Для измерения личностных черт использовался опросник «Большая Пятерка» (форма S методики NEOPI/ NEO-FFI) П. Косты и Р. МакКрэ (Costa, McCrae, 1989) в русской адаптации М.В. Бодунова и С.Д. Бирюкова. Он состоит из 60 пунктов и включает шкалы нейротизма, экстраверсии, открытости опыту, согласия и сознательности.

Процедура. Исследование проводилось индивидуально с каждый испытуемым, сначала предъявлялась методика ШПАНА, потом Большая Пятерка и ЭмИн.

\section{Результаты и обсуждение}

Для оценки внутренней согласованности всех использованных методик использовалась $\alpha$ Кронбаха (таблица 1). Внутренняя согласованность шкал методики ШПАНА очень близка данным авторов оригинальной версии и русской адаптации (0.89 для ПА и 0.86 для НА в обоих случаях). Внутренняя согласованность шкал ЭмИн даже выше, чем в первоначальной публикации опросника, где она находилась в пределах от 0.75 до 0.78. Внутренняя согласованность шкал опросника «Большая Пятерка» удовлетворительна, за исключением шкалы «Открытость опыту» (0.50).

Корреляция между шкалами ПА и НА методики ШПАНА составила $r=-0.40, p<0.001$, что близко тем результатам, которые получили авторы оригинальной версии и русской

Таблица 1

Внутренняя согласованность методик ШПАНА, ЭмИн и Большая Пятерка

\begin{tabular}{|l|l|c|}
\hline \multicolumn{1}{|c|}{ Методика } & \multicolumn{1}{|c|}{ Шкалы } & $\boldsymbol{\alpha}$ Кронбаха \\
\hline \multirow{4}{*}{ ШПАНА } & Шкала позитивного аффекта & 0.86 \\
\cline { 2 - 3 } & Шкала негативного аффекта & 0.88 \\
\hline \multirow{4}{*}{ ЭмИн } & Общий ЭИ & 0.89 \\
\cline { 2 - 3 } & Межличностный ЭИ & 0.86 \\
\cline { 2 - 3 } & Внутриличностный ЭИ & 0.84 \\
\cline { 2 - 3 } & Понимание эмоций & 0.86 \\
\cline { 2 - 3 } & Управление эмоциями & 0.84 \\
\hline \multirow{5}{*}{$\begin{array}{l}\text { Большая пятерка } \\
\text { (NЕО-PI/NEO-FFI) }\end{array}$} & Нейротизм & 0.85 \\
\cline { 2 - 3 } & Экстраверсия & 0.83 \\
\cline { 2 - 3 } & Открытость опыту & 0.50 \\
\cline { 2 - 3 } & Дружелюбие & 0.78 \\
\cline { 2 - 3 } & Сознательность & 0.87 \\
\hline
\end{tabular}


адаптации $(r=-0.41, p<0.001$ и $r=$ $=-0.37, p<0.001$ соответственно).

Методика ШПАНА позволяет также получить показатель аффективного баланса (АБ) для каждого испытуемого. Для этого из балла ПА вычитается балл НА. Показатель АБ позволяет судить о том, в какой степени эмоциональное состояние в целом является положительным или отрицательным (Sheldon et al., 2001; Fernández-Berrocal, Extremera, 2006).

Дальнейшая обработка результатов состояла в корреляционном анализе связей между опросниками, во всех случаях использовались коэффициенты корреляции Пирсона.

В таблице 2 представлены корреляции между настроением и эмоциональным интеллектом испытуемых. Наиболее характерным результатом являются положительные корреляции общего эмоционального интеллекта и двух его компонентов со шкалой ПА и отрицательные корреляции со шкалой НА. Это соответствует результатам других исследований, упомянутых в обзоре литературы. Обращает на себя внимание, что межличностный эмоциональный интеллект и понимание эмоций положительно связаны со шкалой ПА, однако не дают значимых связей со шкалой НА. Этому паттерну соответствуют и корреляции с показателями АБ. Такие результаты позволяют предположить, что в основе связи между эмоциональным интеллектом и настроением лежат регулятивные процессы.

В таблице 3 представлены корреляции между настроением и личностными чертами испытуемых. Как и ожидалось, получена отрицательная связь нейротизма со шкалой ПА и положительная связь со шкалой HА. Экстраверсия положительно связана со шкалой ПА и не имеет связей со шкалой НА. Это в целом соответствует результатам, получаемым другими авторами, за исключением отсутствия связи между экстраверсией и НА.

Для лучшего понимания структуры данных был проведен корреляционный анализ связей между личностными чертами и эмоциональным интеллектом; результаты представлены в таблице 4. Обращают на себя внимание тесные отрицательные

Таблица 2

Корреляции между настроением (методика ШПАНА) и эмоциональным интеллектом (опросник ЭмИн)

\begin{tabular}{|l|c|c|c|}
\hline & Шкала ПА & Шкала НА & АБ \\
\hline Общий ЭИ & $0.38^{* *}$ & $-0.26^{* *}$ & $0.39^{* *}$ \\
\hline Межличностный ЭИ & $0.29^{*}$ & -0.06 & 0.21 \\
\hline Внутриличностный ЭИ & $0.37^{* *}$ & $-0.36^{* *}$ & $0.44^{* *}$ \\
\hline Понимание эмоций & $0.27^{*}$ & -0.08 & 0.21 \\
\hline Управление эмоциями & $0.40^{* *}$ & $-0.36^{* *}$ & $0.45^{* *}$ \\
\hline
\end{tabular}

${ }^{*} p<0.05,{ }^{* *} p<0.01$. 
Корреляции между настроением (методика ШПАНА) и личностными чертами (опросник «Большая Пятерка»)

\begin{tabular}{|l|c|c|c|}
\hline & Шкала ПА & Шкала НА & АБ \\
\hline Нейротизм & $-0.39^{* *}$ & $0.49^{* *}$ & $-0.52^{* *}$ \\
\hline Экстраверсия & $0.29^{*}$ & -0.08 & 0.23 \\
\hline Открытость опыту & 0.12 & 0.02 & 0.06 \\
\hline Дружелюбие & 0.02 & -0.17 & 0.10 \\
\hline Сознательность & 0.18 & -0.02 & 0.13 \\
\hline
\end{tabular}

${ }^{*} p<0.05,{ }^{* *} p<0.01$.

Таблица 4

Корреляции между эмоциональным интеллектом (опросник ЭмИн) и личностными чертами (опросник «Большая Пятерка»)

\begin{tabular}{|l|c|c|c|c|c|}
\hline & Общий ЭИ & МЭИ & ВЭИ & ПЭ & УЭ \\
\hline Нейротизм & $-0.68^{* *}$ & $-0.49^{* *}$ & $-0.66^{* *}$ & $-0.43^{* *}$ & $-0.74^{* *}$ \\
\hline Экстраверсия & 0.22 & $0.37^{* *}$ & 0.05 & 0.21 & 0.19 \\
\hline Открытость опыту & 0.16 & 0.21 & 0.07 & 0.16 & 0.12 \\
\hline Дружелюбие & $0.24^{*}$ & $0.28^{*}$ & 0.15 & $0.24^{*}$ & 0.18 \\
\hline Сознательность & $0.50^{* *}$ & $0.36^{* *}$ & $0.50^{* *}$ & $0.40^{* *}$ & $0.48^{* *}$ \\
\hline
\end{tabular}

${ }^{*} p<0.05,{ }^{* *} p<0.01$.

связи между нейротизмом и всеми показателями эмоционального интеллекта, а также достаточно высокие положительные связи между сознательностью и эмоциональным интеллектом. Применение методологии линейно-структурного моделирования позволило бы выявить латентные факторы, стоящие за этими корреляциями. Недостаточный объем выборки (71 испытуемый) пока не позволяет этого сделать, но в дальнейшем такой анализ представляется необходимым.

Таким образом, мы впервые получили результаты, описывающие связь настроения с личностными чертами и эмоциональным интеллек- том на русскоязычных испытуемых. Необходимо отметить некоторые ограничения настоящего исследования. Во-первых, использованная методика ШПАНА описывает настроения только по двум измерениям. Инструменты, допускающие более дифференцированное описание настроений, позволили бы получить более тонкие результаты. Пока методик такого рода с удовлетворительными психометрическими характеристиками на русском языке не существует, но в перспективе их применение было бы плодотворно. Во-вторых, использовались только самоотчетные методики. Хорошо известно, что самоотчеты испытуемых 
относительно своего эмоционального состояния положительно коррелируют с их самоотчетами относительно своих эмоциональных личностных черт (Brose et al., 2013). Возможно, этим частично обусловлены полученные корреляции с нейротизмом и экстраверсией. Представляется более продуктивным использовать не одноразовый замер настроения, но собирать данные о настроении человека на протяжении некоторого времени (так называе- мый experience sampling). Это позволит получить более достоверные сведения об эмоциональном фоне, характерном для того или иного испытуемого, и проанализировать связи между личностными чертами и эмоциональным интеллектом, с одной стороны, и динамическими характеристиками эмоциональных состояний, с другой стороны. Такие данные смогут пролить свет на роль регулятивных процессов, связывающих личность и настроение.

\section{Литература}

Берри, Дж. В., Пуртинга, А. Х., Сигалл, М. Х., Дасен, П. Р. (2007). Кросс-культурная психология. Исследования и применение. Харьков: Гуманитарный центр.

Люсин, Д. В. (2009). Опросник на эмоциональный интеллект ЭмИн: новые психометрические данные. В кн. Д. В. Люсин, Д. В. Ушаков (Ред.), Социальный и эмоциональный интеллект: от моделей к измерениям (с. 264-278). М.: Изд-во «Институт психологии РАН».

Люсин, Д. В. (2014). Опыт разработки самоотчетной методики для оценки эмоциональных состояний. В кн. В. Т. Кудрявцев (Ред.), Мьиление и речь: подходы, проблемъ, решения. Материалы XV Международных чтений памяти Л.С. Выготского (т. 1, с. 140-143). М.: Левъ.

Осин, Е. Н. (2012). Шкалы позитивного и негативного аффекта (ШПАНА): разработка русскоязычного аналога методики PANAS. Психология. Журнал Высшей школы экономики, 9(4), 91-110.

Рубинштейн, С. Л. (1946). Основы общей психологии. М..

Brose, A., Lindenberger, U., \& Schmiedek, F. (2013). Affective states contribute to trait reports of affective well-being. Emotion, 13, 940-948.

Costa, P. T., \& McCrae, R. R. (1989). The NEO-PI/NEO-FFI manual supplement. Odessa, FL: Psychological Assessment Resources.

David, J., Green, P., Martin, R., \& Suls, J. (1997). Differential roles of neuroticism, extraversion and event desirability on mood in daily life: An integrative model of top-down and bottom-up influences. Journal of Personality and Social Psychology, 73, 149-159.

Extremera, N., \& Fernández-Berrocal, P. (2005). Perceived emotional intelligence and life satisfaction: predictive and incremental validity using the Trait Meta-Mood Scale. Personality and Individual Differences, 39, 937-948.

Feldman Barrett, L., \& Russell, J. A. (1999). The structure of current affect: controversies and emerging consensus. Current Directions in Psychological Science, 8, 10-14.

Fernández-Berrocal, P., \& Extremera, N. (2006). Emotional intelligence and emotional reactivity and recovery in laboratory context. Psichothema, 18, 72-78.

Frijda, N. H. (1993). Moods, emotion episodes, and emotions. In M. Lewis \& J. M. Haviland (Eds.), Handbook of emotions (pp. 381-403). New York: Guilford Press.

McCrae, R. R., \& Costa, P. T. (2005). Personality in adulthood. New York: Guilford Press. 
Palmer, B. R., Donaldson, C., \& Stough, C. (2002). Emotional intelligence and life satisfaction. Personality and Individual Differences, 33, 1091-1100.

Salovey, P., Mayer, J. D., Goldman, S. L., Turvey, C., \& Palfai, T. P. (1995). Emotional attention, clarity and repair: exploring emotional intelligence using the Trait Meta-Mood Scale. In J. W. Pennebaker (Ed.), Emotion, disclosure and health (pp. 125-154). Washington, DC: American Psychological Association.

Schimmack, U., \& Grob, A. (2000). Dimensional models of core affect: a quantitative comparison by means of structural equation modeling. European Journal of Personality, 14, 325-345.

Schutte, N. S., Malouff, J., Simunek, M., Hollander, S., \& McKenley, J. (2002). Characteristic emotional intelligence and emotional well-being. Cognition and Emotion, 16, 769-785.

Sheldon, K.M., Elliot, A.J., Kim, Y., \& Kasser, T. (2001). What's satisfying about satisfying events? Comparing ten candidate psychological needs. Journal of Personality and Social Psychology, 54, 1063-1070.

Velting, D. M. (1999). The effectiveness of coping meta-strategies: perceived efficiency, emotional correlates and cognitive performance. Personality and Individual Differences, 26, 923-939.

Watson, D., \& Clark, L. A. (1992). On traits and temperament: general and specific factors of emotional experience and their relation to the five-factor model.Journal of Personality, 60, 441-476.

Watson, D., Clark, L. A., \& Tellegen, A. (1988). Development and validation of brief measures of positive and negative affect: The PANAS scales. Journal of Personality and Social Psychology, 54, 1063-1070.

Wundt, W. (1896). Grundriss der Psychologie. Leipzig: Engelmann. (in German).

Люсин Дмитрий Владимирович, ведущий научный сотрудник НИУ ВНЭ; стариий научный сотрудник Института психологии РАН, кандидат педагогических наук, доцент

Контакты: ooch@mail.ru

Овсянникова Виктория Владимировна, старший научный сотрудник НИУ ВНЭ, кандидат психологических наук, доцент

Контакты: v.ovsyannikova@gmail.com

\title{
Relationships between Emotional Intelligence, Personality Traits and Mood
}

\author{
Dmitry V. Lyusin \\ Leading Research Fellow, HSE*; Senior Research Fellow, Institute of Psychology of Russian \\ Academy of Sciences**, Ph.D. \\ E-mail: ooch@mail.ru \\ Viktoria V. Ovsyannikova \\ Senior Research Fellow, HSE*, Ph.D. \\ E-mail: v.ovsyannikova@gmail.com \\ Address: *20 Myasnitskaya str., Moscow, 101000, Russian Federation \\ ** 13 build.1 Yaroslavskaya str., Moscow, 123366, Russian Federation
}




\begin{abstract}
The main objective of the study is the search of relationships between mood, personality traits and emotional intelligence (EI). The additional goal is acquisition of the new psychometric data on the used questionnaires. To measure mood, the Russian adaptation of the questionnaire PANAS was used, which assesses mood by the scales of positive affect (PA) and negative affect (NA). Personality traits were measured with the help of the Big 5 questionnaire, emotional intelligence was measured with the help of the questionnaire EmIn. Questionnaires were used on a sample of 71 subjects aged 18-49 years. Data was received about the reliability of the scales of all the used questionnaires, which in whole was satisfactory - Chronbach's $\alpha$ of all the scales except one lie in the range from .78 to .89. Positive correlations of some scales of the EmIn questionnaire (general EI, interpersonal EI, management of emotions) were found with the scale PA and negative correlations were found with the scale NA. Scales of interpersonal EI and understanding of emotions had only positive correlations with the scale PA. Negative correlation of neuroticism with the scale PA and positive correlation with the scale NA were found. Extraversion was positively correlated with the scale PA and had no correlations with the scale NA. In whole the acquired results correspond to the results of the similar studies, conducted in other countries. The necessity to move from one-time measurements to data collection about participants' mood for a long period of time (the so-called experience sampling) is validated. It will allow acquiring more reliable information about emotional background, characteristic for this or that participant, and analyzing relationships between personality traits and emotional intelligence, from the one side, and dynamic characteristics of emotional states, from the other side.
\end{abstract}

Keywords: mood, emotional intelligence, big five, personality traits.

\title{
References
}

Berry, J. W., Poortinga, Y. H., Segall, M. H., \& Dasen, P. R. (2007). Kross-kul'turnaya psikhologiya. Issledovaniya i primenenie [Cross-cultural psychology. Research and applications]. Kharkiv: Gumanitarnyi Tsentr. (Transl. of: Berry, J. W., Poortinga, Y. H., Segall, M. H., \& Dasen, P. R. (2002). Cross-cultural psychology: Research and applications (2nd ed.). Cambridge, UK: Cambridge Unversity Press).

Brose, A., Lindenberger, U., \& Schmiedek, F. (2013). Affective states contribute to trait reports of affective well-being. Emotion, 13, 940-948.

Costa, P. T., \& McCrae, R. R. (1989). The NEO-PI/NEO-FFI manual supplement. Odessa, FL: Psychological Assessment Resources.

David, J., Green, P., Martin, R., \& Suls, J. (1997). Differential roles of neuroticism, extraversion and event desirability on mood in daily life: An integrative model of top-down and bottom-up influences. Journal of Personality and Social Psychology, 73, 149-159.

Extremera, N., \& Fernández-Berrocal, P. (2005). Perceived emotional intelligence and life satisfaction: predictive and incremental validity using the Trait Meta-Mood Scale. Personality and Individual Differences, 39, 937-948. 
Feldman Barrett, L., \& Russell, J. A. (1999). The structure of current affect: controversies and emerging consensus. Current Directions in Psychological Science, 8, 10-14.

Fernández-Berrocal, P., \& Extremera, N. (2006). Emotional intelligence and emotional reactivity and recovery in laboratory context. Psichothema, 18, 72-78.

Frijda, N. H. (1993). Moods, emotion episodes, and emotions. In M. Lewis \& J. M. Haviland (Eds.), Handbook of emotions (pp. 381-403). New York: Guilford Press.

Lyusin, D. V. (2009). Oprosnik na emotsional'nyi intellekt EmIn: novye psikhometricheskie dannye [Questionnaire on emotional intelligence EmIn: New psychometric data]. In D. V. Lyusin \& D. V. Ushakov (Eds.), Sotsial'nyi i emotsional'nyi intellekt: ot modelei $k$ izmereniyam [Social and emotional intelligence: From models to measurements] (pp. 264-278). Moscow: Institute of Psychology of Russian Academy of Sciences.

Lyusin, D. V. (2014). Opyt razrabotki samootchetnoi metodiki dlya otsenki emotsional'nykh sostoyanii [Development experience of a self-report scale for evaluation of emotional states]. In V. T. Kudryavtsev (Ed.), Myshlenie i rech': podkhody, problemy, resheniya. Materialy XV Mezhdunarodnykh chtenii pamyati L.S. Vygotskogo [Thinking and speech: Approaches, problems, solutions. Materials of the XV International readings in memory of L.S. Vygotsky] (Vol. 1, pp. 140-143). Moscow: Lev.

McCrae, R. R., \& Costa, P. T. (2005). Personality in adulthood. New York: Guilford Press.

Osin, E. (2012). Measuring positive and negative affect: Development of a Russian-language analogue of PANAS . Psychology. Journal of the Higher School of Economics, 9(4), 91-110.

Palmer, B. R., Donaldson, C., \& Stough, C. (2002). Emotional intelligence and life satisfaction. Personality and Individual Differences, 33, 1091-1100.

Rubinshtein, S. L. (1946). Osnovy obshchei psikhologii [Basics of general psychology]. Moscow.

Salovey, P., Mayer, J. D., Goldman, S. L., Turvey, C., \& Palfai, T. P. (1995). Emotional attention, clarity and repair: exploring emotional intelligence using the Trait Meta-Mood Scale. In J. W. Pennebaker (Ed.), Emotion, disclosure and health (pp. 125-154). Washington, DC: American Psychological Association.

Schimmack, U., \& Grob, A. (2000). Dimensional models of core affect: a quantitative comparison by means of structural equation modeling. European Journal of Personality, 14, 325-345.

Schutte, N. S., Malouff, J., Simunek, M., Hollander, S., \& McKenley, J. (2002). Characteristic emotional intelligence and emotional well-being. Cognition and Emotion, 16, 769-785.

Sheldon, K.M., Elliot, A.J., Kim, Y., \& Kasser, T. (2001). What's satisfying about satisfying events? Comparing ten candidate psychological needs. Journal of Personality and Social Psychology, 54, 1063-1070.

Velting, D. M. (1999). The effectiveness of coping meta-strategies: perceived efficiency, emotional correlates and cognitive performance. Personality and Individual Differences, 26, 923-939.

Watson, D., \& Clark, L. A. (1992). On traits and temperament: general and specific factors of emotional experience and their relation to the five-factor model. Journal of Personality, 60, 441-476.

Watson, D., Clark, L. A., \& Tellegen, A. (1988). Development and validation of brief measures of positive and negative affect: The PANAS scales. Journal of Personality and Social Psychology, 54, 1063-1070.

Wundt, W. (1896). Grundriss der Psychologie. Leipzig: Engelmann. (in German). 